\title{
A study of the effectiveness of direct access day case oral surgery compared with standard letter referral
}

\author{
Direct access day case oral surgery A. Joshi, L. Doyle, H. V. Worthington, and J. P. Rood, Br Dent J 2000; 188: 452-456
}

\author{
Objective \\ To compare the effectiveness of direct access referrals with \\ standard letter referrals, and also assess the impact of the direct \\ access system on the primary care provider, secondary sector and \\ from the patients perspective.
}

\section{Design}

Surgical and anaesthetic guidelines were agreed and 12 general dental practitioners were recruited to participate in the study. Each practitioner was given 100 envelopes which contained randomly allocated 50 direct and 50 standard referrals. For direct referrals, GDPs completed a pre-operative assessment and obtained an operation date by telephone from the day case unit while the patient was in the surgery. The clinical history sheet was faxed to the day unit and the radiographs posted. The only contact the patient had with the hospital was on the day of surgery. Standard referral patients were referred in the traditional way with a referral letter being sent to an out-patient clinic. Evaluation of the effectiveness of the direct referral system versus standard letter method were made via completion of questionnaires by the patients, dental practitioners, hospital clinicians, day case anaesthetist and co-ordinator.

\section{Setting}

The study was carried out over 2 years commencing in 1997 at the Oral Surgery Day Case Unit at Manchester Royal Infirmary.

\section{Results}

A greater number of direct access referrals (409; 90\%) were treated in comparison with $(312 ; 75 \%)$ standard referrals
$(P<0.001)$. Eighty nine per cent of direct access records were adequate pre-operatively. More than $70 \%$ of dentists and hospital clinicians preferred the direct access referral method. Sixty-three per cent of standard letter patients were satisfied with their mode of referral compared with $87 \%$ of direct access patients.

\section{Conclusions}

Given appropriate guidelines dental practitioners are able to refer directly to oral surgery day case operation lists. This has proved to be the favoured method of referral for dental practitioners, the secondary sector and patients.

\section{In brief}

- Twelve general dental practitioners were invited to participate in a prospective study to compare the effectiveness of direct access referrals with standard letter referrals for routine dento-alveolar surgery.

- Given appropriate surgical and anaesthetic guidelines, dental practitioners are able to refer directly to oral surgery day case operation lists.

- There was good collaborative team work between the primary and secondary sector.

- The direct access referral method was preferred by GDPs, hospital clinicians and patients. This resulted in fewer visits to the hospital and shorter waiting times for patients. A greater number of direct access referrals were treated compared with standard letter referrals.

\section{Comment}

Direct access referrals for dento-alveolar were received from 12 specially recruited GDPs, all of whom had access to pre-packaged referral documents (including specific patient selection criteria), haemoglobin, blood pressure and weight monitoring equipment, and fax machine access to the Oral Day Case Unit at Manchester Royal Infirmary.

The ability to diagnose, assess the suitability for and co-ordinate hospital operating dates for patients presenting with oral surgical conditions in general dental practice offers a number of possible advantages for both patients and practitioners: fewer hospital attendances prior to surgery, immediate confirmation of operation dates, and a reduction in waiting times.

In this study, $90 \%$ of direct access referrals were successfully treated compared with $75 \%$ of standard referrals (in which tradi- tional referral letters initiated standard hospital out-patient consultation procedures). The most common reasons for failure to treat in both groups, however, remained patients who either did not attend on the day or who cancelled their appointments. In a smaller number of cases the proposed treatment was deemed inappropriate or the patient was unfit for day case surgery.

The majority of GDPs, hospital clinicians and patients involved expressed considerable support for the direct access system, although potential disadvantages include the lack of access to a specialist opinion and discussion of treatment options prior to surgery and the loss of the 'validation' role of out-patient attendances, both of which may help reduce patient failures.

A successful alternative model uses nurse-led pre-admission clinics where, following intitial hospital consultation, patients attend $2-4$ weeks prior to pro- posed surgical admission. This facilates clinical review of the patient, medical screening and health education, allows discussion with an 'independent' health professional, and by encouraging proactive patient involvement with date selection, minimises failures and cancellations on the day of surgery.

Direct access referral systems inevitably transfer both clinical responsibilities and financial and administrative burdens to the primary care. Further research is needed to determine the feasability of involving greater numbers of GDPs in direct access day surgery, and the practicality of ensuring appropriate funding for their extended role.

\section{P J Thomson}

Professor of Oral \& Maxillofacial Surgery, The Dental School, University of Newcastle upon Tyne 